\title{
Neural Network Ensemble with Negatively Correlated Features for Cancer Classification
}

\author{
Hong-Hee Won and Sung-Bae Cho \\ Dept. of Computer Science, Yonsei University, 134 Shinchon-dong, Sudaemoon-ku, \\ Seoul 120-749, Korea \\ cool@candy.yonsei.ac.kr, sbcho@cs.yonsei.ac.kr
}

\begin{abstract}
The development of microarray technology has supplied a large volume of data to many fields. In particular, it has been applied to prediction and diagnosis of cancer, so that it expectedly helps us to exactly predict and diagnose cancer. It is essential to efficiently analyze DNA microarray data because the amount of DNA microarray data is usually very large. Since accurate classification of cancer is very important issue for treatment of cancer, it is desirable to make a decision by combining the results of various expert classifiers rather than by depending on the result of only one classifier. In spite of many advantages of ensemble classifiers, ensemble with mutually errorcorrelated classifiers has a limit in the performance. In this paper, we propose the ensemble of neural network classifiers learned from negatively correlated features to classify cancer precisely, and systematically evaluate the performance of the proposed method using three benchmark datasets. Experimental results show that the neural network ensemble with negatively correlated features produces the best recognition rate on the three benchmark datasets.
\end{abstract}

\section{Introduction}

DNA microarray technology has advanced so much that we can simultaneously measure the expression levels of thousands of genes under particular experimental environments and conditions [1]. DNA microarray technology makes it possible to understand life on the molecular level. The development of DNA microarray technology enables to generate large-scale gene expression data. It has led to many statistical and analytical challenges from the problems in biology because it has been produced large amount of genes. We can analyze the gene information very rapidly and precisely by managing them at one time using several statistical methods and machine learning.

Cancer classification in clinical practice relied on clinical and histopathological information can be often incomplete or misleading. DNA microarray technology has been applied to the field of accurate prediction and diagnosis of cancer and expected that it would help them. Molecular level diagnostics with gene expression profiles can offer the methodology of precise, objective, and systematic cancer classification. Especially accurate classification of cancer is very important issue for treatment of cancer. Since the gene expression data usually consist of huge number of genes, 
several researchers have been studying many problems of cancer classification using data mining methods, machine learning algorithms and statistical methods to efficiently analyze these data [2, 3]. However, most researchers partly have evaluated only the performance of the feature selection method and classifier.

In this paper, we attempt to use the negative correlation of the features. We define two ideal feature vectors for a standard of good feature, and utilize the features selected by scoring the similarity with each ideal feature vector. Two ideal feature vectors are the one high in class A and low in class B, and the other one low in class A and high in class B. Since the vectors have negative correlation, the sets of genes similar to each ideal vector are also negatively correlated. The negatively correlated features represent two different aspects of classification boundary for gene expression data. We can search in a much wider solution space by combining these features. In this paper, we propose the ensemble classifier trained with negatively correlated features. We test the proposed method in three benchmark cancer datasets, and systematically analyze the usefulness of the negative correlation.

\section{DNA Microarray}

DNA arrays consist of a large number of DNA molecules spotted in a systemic order on a solid substrate. Depending on the size of each DNA spot on the array, DNA arrays can be categorized as microarrays when the diameter of DNA spot is less than 250 microns, and macroarrays when the diameter is bigger than 300 microns. The arrays with the small solid substrate are also referred to as DNA chips. It is so powerful that we can investigate the gene information in short time, because at least hundreds of genes can be put on the DNA microarray to be analyzed.

There are two representative DNA microarray technologies: cDNA microarray technology and oligonucleotide microarray technology. cDNA microarrays are composed of thousands of individual DNA sequences printed in a high density array on a glass microscope slide using a robotic arrayer. High-density oligonucleotide microarrays are made using spatially patterned, light-directed combinatorial chemical synthesis, and contain up to hundreds of thousands of different oligonucleotides on a small glass surface. For mRNA samples, the two samples are reverse-transcribed into cDNA, labeled using different fluorescent dyes mixed (red-fluorescent dye Cy5 and green-fluorescent dye Cy3). After the hybridization of these samples with the arrayed DNA probes, the slides are imaged using scanner that makes fluorescence measurements for each dye. The log ratio between the two intensities of each dye is used as the gene expression data.

$$
\text { gene_expression }=\log _{2} \frac{\operatorname{Int}(\mathrm{Cy} 5)}{\operatorname{Int}(\mathrm{Cy} 3)}
$$

where $\operatorname{Int}(\mathrm{Cy} 5)$ and $\operatorname{Int}(\mathrm{Cy} 3)$ are the intensities of red and green colors. Since at least hundreds of genes are put on the DNA microarray, we can investigate the genomewide information in short time. 


\section{Neural Network Ensemble with Informative Features}

We propose the ensemble classifier with mutually exclusive features and negatively correlated features. The framework of the proposed ensemble classifier with mutually exclusive features and negatively correlated features is shown in Figure 1. The basic idea of ensemble classifier scheme is to develop several pairs of trained neural networks with feature sets selected on correlation analysis, and to classify a given input pattern by utilizing combination methods. Then it naturally raises the question of obtaining a consensus on the results of each individual network.

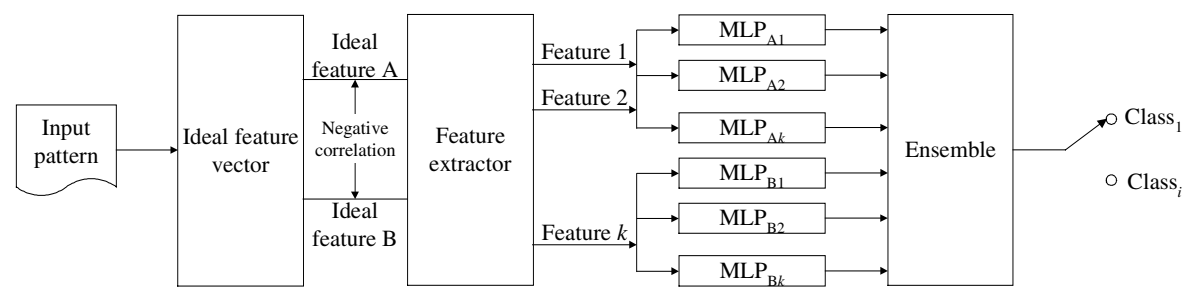

Fig. 1. Overview of the ensemble classifier with negatively correlated features

\subsection{Neural Network Ensemble with Mutually Exclusive Features}

Among thousands of genes whose expression levels are measured, not all are needed for classification. Microarray data consist of large number of genes in small samples. For efficient classification, we need to find out the informative features from input observation. This process is referred to as gene selection [4].

Suppose that we have a $M \times N$ training set where $M$ is the number of samples (input vector) and $N$ is the number of features (dimensionality of input vector). The $i$ th feature of samples, $g_{i}$, can be expressed:

$$
g_{i}=\left(e_{1}, e_{2}, e_{3}, \cdots, e_{M}\right)
$$

where $e$ is the data and $i=1 \sim N$. We want to know the locations of informative $k$ features out of $M$. Suppose $g_{\text {ideal }}$ is an ideal vector representing class $c_{j}$. If it is possible to know representative vector $g_{\text {ideal }}$ for class $c_{j}$, we can simply measure the correlation and similarity of $g_{i}$ to classes, which tells the feature-goodness. Modeling $g_{\text {ideal }}$, we should use prior knowledge and intuitional experience about classes.

$$
g_{\text {ideal }}=\left(e_{1}, e_{2}, e_{3}, \cdots, e_{M}{ }^{\prime}\right)
$$

Measuring the similarity of $g_{i}$ and $g_{\text {ideal }}$ using similarity measures, Pearson's correlation coefficient $(P C)$, Spearman coefficient $(S C)$, Euclidean distance $(E D)$, and cosine coefficient $(C C)$ [4], the most similar 25 genes are used for classification. Also, the most informative 25 genes can be selected using information-theoretic feature selection methods, information gain $(I G)$, mutual information $(M I)$, and signal to noise ratio [5]. Using feature selection, we get a set of informative features from the data. In order to choose mutually exclusive features, we have plotted the distribution of $g_{i}$ from two feature selection methods. If the two features are mutually 
exclusive, the distribution will be in the (-) direction, otherwise (+) direction. The mutually exclusive feature is to encourage set of classifiers to learn different aspects of data.

We have used multi-layer perceptron (MLP), self-organizing map (SOM), structure adaptive SOM (SASOM), support vector machine (SVM), decision tree (DT) and $k$ nearest neighbor (KNN) as classifiers [5]. For combining classifiers, a neural network and majority voting are used. A neural network combines the outputs of single classifiers in our system. Outputs of single classifiers can be thought of as CSVs (classification status values), which contain information on answer patterns of classifiers. Neural network has $m+n$ (dimensionality of CSV) input nodes and $j$ output nodes.

\subsection{Neural Network Ensemble with Negatively Correlated Features}

To select negatively correlated features, we define two ideal feature vectors as the one high in class $A$ and low in class $B(1,1, \ldots, 1,0,0, \ldots, 0)$, and the other one low in class $A$ and high in class $B(0,0, \ldots, 0,1,1, \ldots, 1)$ as shown in Figure 2 and select the sets of informative genes with high similarity to each ideal gene vector. Since Pearson's correlation coefficient of two ideal gene vectors is -1 , two vectors are perfectly negative-correlated. The sets of gene vectors are also highly negative-correlated. Correlation analysis and distance measure methods are used in order to measure the similarity of gene vector $g_{i}$ and ideal gene vectors A and B. Similarity measures used for negative correlation are Pearson correlation coefficient, Spearman correlation coefficient, Euclidean distance, and cosine coefficient [5]. The informative features selected by negative correlation represent two different aspects of training data. We can search in a much wider solution space by combining these features.

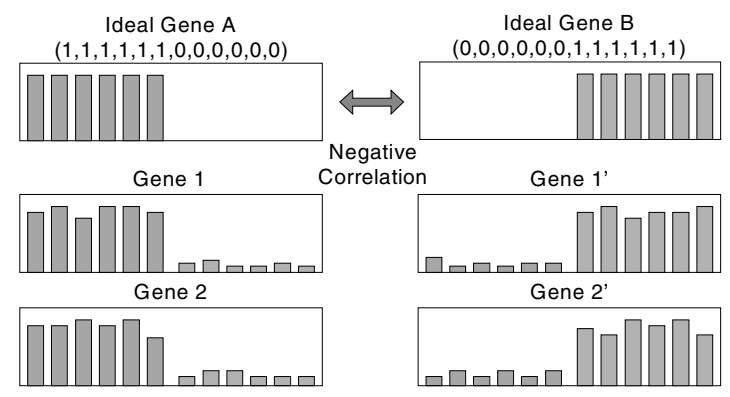

Fig. 2. Informative genes selected using negative correlation

We have chosen multi-layer perceptron for classification and the Bayesian approach for final decision of the neural network classifier because the Bayesian approach can solve the problem of tie-break in ensemble by using a priori knowledge of each combined classifier. The Bayesian approach combines classifiers with different weights by using the previous knowledge of each classifier. Where $k$ classifiers are combined, $c_{i}, i=1, \ldots, m$, is the class of a sample, classifier $\left._{j}\right)$ is the class 
of the $j$ th classifier, and $w_{i}$ is a priori possibility of the class $c_{i}$, Bayesian combination is defined as follows:

$$
\left.c_{\text {ensemble }}=\underset{1 \leq i \leq m}{\arg \max }\left\{w_{i} \prod_{j=1}^{k} P\left(c_{i} \mid \text { c }_{\text {(assifier }}\right)\right)\right\}
$$

\section{Experiments}

\subsection{Datasets}

Three representative datasets, leukemia cancer dataset, colon cancer dataset and lymphoma cancer dataset, are used in this paper among several microarray datasets. Leukemia dataset consists of 72 samples: 25 samples of acute myeloid leukemia (AML) and 47 samples of acute lymphoblastic leukemia (ALL). 38 out of 72 samples were used as training data and the remaining were used as test data. Each sample contains 7129 gene expression levels. Colon dataset consists of 62 samples of colon epithelial cells taken from colon-cancer patients. Each sample contains 2000 gene expression levels. 31 out of 62 samples were used as training data and the remaining were used as test data. Lymphoma dataset consists of 24 samples of GC B-like and 23 samples of activated B-like. 22 out of 47 samples were used as training data and the remaining were used as test data.

\subsection{Results Analysis of Ensemble with Mutually Exclusive Features}

The results of recognition rate on the test data with single feature and classifier are as shown in Table 1. The MLP seems to have the best recognition rate among the classifiers on the average. $97.1 \%$ of accuracy is the best throughout all the classifiers and features. The performance of classifiers seems to be somewhat dependent on the feature it uses. SVM, for example, has $97.1 \%$ of accuracy with Pearson's correlation, but $58.8 \%$ with information gain and mutual information.

Table 1. Recognition rates by feature and classifier (\%)

\begin{tabular}{cccccc}
\hline & MLP & SOM & SVM & DT & KNN \\
\hline PC & 97.1 & 74.1 & 97.1 & 97.1 & 88.2 \\
SC & 70.6 & 67.4 & 70.6 & 61.8 & 73.5 \\
ED & 97.1 & 70.6 & 91.2 & 82.4 & 82.4 \\
CC & 79.4 & 70.6 & 70.6 & 73.5 & 76.5 \\
IG & 72.9 & 63.8 & 58.8 & 47.1 & 70.6 \\
MI & 62.1 & 68.8 & 58.8 & 55.9 & 58.8 \\
SN & 94.1 & 97.1 & 94.1 & 91.2 & 94.1 \\
\hline
\end{tabular}


Figure 3 illustrates the dependency between two feature selection methods. In case (a), dots are distributed in negative direction. Therefore, the feature sets chosen by Pearson's correlation and Euclidean distance must be very mutually exclusive, and the classifiers with these feature selection methods are trained in independent feature spaces each other. In case (b), dots are distributed in a triangular form. We cannot explicitly see the direction of the correlation. In case (c), genes selected by one method with high score also appear in the list of top-ranking genes by the other method. Since two sets of classifiers are trained in mutually dependent feature spaces, it is hard to expect the performance improvement when the classifiers are combined.

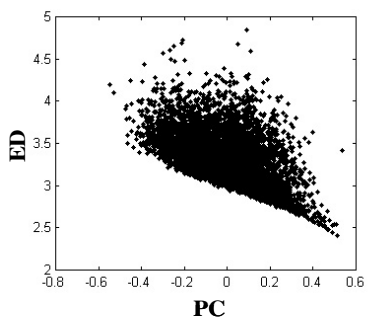

(a) Negative correlation (coefficient: $\mathbf{- 0 . 5 2}$ )

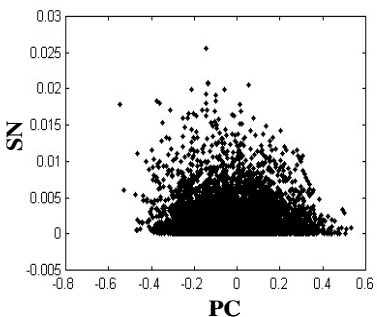

(b) Neutral (coefficient: $\mathbf{- 0 . 0 3 )}$

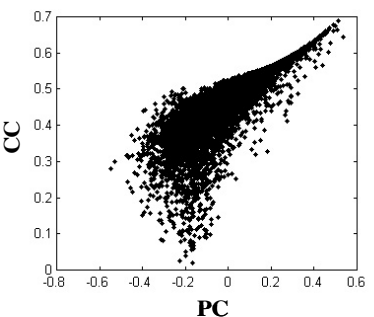

(c) Positive correlation (coefficient: $\mathbf{0 . 8 0}$ )

Fig. 3. Correlations of feature selection methods in Leukemia

Table 2 is the result of the ensemble classifiers. Case (a), (b) and (c) are investigated and we also have combined all the features, for the comparison with others. Case (a) produces the best recognition rates, $97.1 \%$ in both neural network and voting method, which is also the best that we could get in the experiments with single feature/classifier. Case (b) also produces relatively high rates. Case (c) and 'all feature,' however, turn to be bad, which implies that combining independent features is efficient, producing much higher performance than when all features are considered.

Table 2. Results of ensemble classifiers in Leukemia (\%)

\begin{tabular}{ccc}
\hline & Neural network & Majority voting \\
\hline Case (a) & 97.1 & 97.1 \\
Case (b) & 97.1 & 94.1 \\
Case (c) & 91.2 & 85.3 \\
All features & 64.7 & 41.2 \\
\hline
\end{tabular}

\subsection{Results Analysis of Ensemble with Negatively Correlated Features}

Table 3 shows the recognition rate of the basis classifiers in each dataset. In Leukemia dataset, MLP I with Pearson's correlation coefficient and MLP I with information 
gain produce the best recognition rate, 97.1\%, among the feature-classifier combinations. In Colon dataset, MLP I with cosine coefficient produces the best recognition rate, 83.9\%. In Lymphoma dataset, MLP II with Spearman's correlation coefficient produces the best recognition rate, $88.0 \%$. While MLP I outperforms MLP II in Leukemia dataset and Colon dataset, MLP II outperforms MLP I in Lymphoma dataset.

Table 3. Recognition rate with features and classifiers (\%)

\begin{tabular}{ccccccc}
\hline & \multicolumn{2}{c}{ Leukemia } & \multicolumn{2}{c}{ Colon } & \multicolumn{2}{c}{ Lymphoma } \\
\cline { 2 - 7 } & MLP I & MLP II & MLP I & MLP II & MLP I & MLP II \\
\hline PC & 97.1 & 79.4 & 74.2 & 77.4 & 64.0 & 72.0 \\
SC & 82.4 & 79.4 & 58.1 & 64.5 & 60.0 & 88.0 \\
ED & 91.2 & 61.8 & 67.8 & 77.4 & 56.0 & 72.0 \\
CC & 94.1 & 76.5 & 83.9 & 77.4 & 68.0 & 76.0 \\
\hline Mean & 91.2 & 74.3 & 71.0 & 74.2 & 62.0 & 77.0 \\
\hline
\end{tabular}

Figure 4 shows the average and the best recognition rates of the ensemble classifiers for Lymphoma dataset. In case of the negatively correlated feature set (MLP I + MLP II), 8 diverse feature sets have been produced with 2 ideal feature vectors (Ideal Gene A and Ideal Gene B) and 4 similarity measures. The classifiers learned with 8 diverse feature sets have been combined using Bayesian approach. The average recognition rate means the average of all possible ${ }_{8} C_{k}(k=2,3$ and 4) combinations of ensemble classifiers.

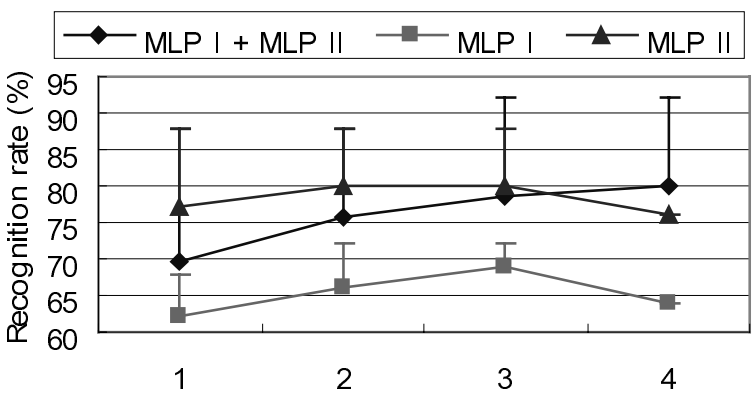

The number of combined classifiers

Fig. 4. Recognition rate of the ensemble in Lymphoma

The performance of the ensemble classifier is superior to the basis in all benchmark datasets. The best recognition rate of ensemble classifier is $97.1 \%$ in Leukemia dataset, $87.1 \%$ in Colon dataset, and $92.0 \%$ in Lymphoma dataset. Compared with the best recognition rates of base classifiers, $97.1 \%, 83.9 \%$, and $92.0 \%$ on the datasets respectively in Table 3, the performance of ensemble is better.

Compared with the results of MLP I and MLP II, the negatively correlated features set (MLP I + MLP II) does not outperform in the average recognition rate, but 
outperforms in the best recognition rate. While the best recognition of the ensemble of MLP I and MLP II is decreasing as the number of combined classifiers is increasing, the best recognition of the ensemble of the negatively correlated coefficient feature set is increasing.

\section{Concluding Remarks}

In order to predict the cancer class of patients, we have illustrated a classification framework that combines sets of classifiers using the correlation information. The results clearly show that the suggested framework works and we may improve the classification performance by combining mutually exclusive sets of classifiers learned from two independent features and by combining independent sets of classifiers learned from negatively correlated features, even when we use simple combination method of voting and Bayesian approach.

The experimental results also imply some correlations between features, which might guide the researchers to choose or devise the best ensemble classifier for their problems in bioinformatics. Based on the results, we have developed the optimal feature-classifier combination to produce the best performance on the classification.

Moreover, the neural network classifier with negative correlation outperforms the ensemble classifiers without negative correlation. We have confirmed that mutually exclusiveness and the negative correlation enable the ensemble classifier to work better by providing appropriate information for the classification to classifiers

Acknowledgements. This work was supported by Biometrics Engineering Research Center and a grant of Korea Health 21 R\&D Project, Ministry of Health \& Welfare, Republic of Korea.

\section{References}

[1] Harrington, C. A., Rosenow, C., and Retief, J., "Monitoring gene expression using DNA microarrays," Curr. Opin. Microbiol., vol. 3, pp. 285-291, 2000.

[2] Ben-Dor, A., Bruhn, L., Friedman, N., Nachman, I., Schummer, M. and Yakhini, N., "Tissue classification with gene expression profiles," Journal of Computational Biology, vol. 7, pp. 559-584, 2000.

[3] Dudoit, S., Fridlyand, J. and Speed, T. P., "Comparison of discrimination methods for the classification of tumors using gene expression data," Technical Report 576, Department of Statistics, University of California, Berkeley, 2000.

[4] Li, L., Weinberg, C. R., Darden, T. A. and Pedersen, L. G., "Gene selection for sample classification based on gene expression data: Study of sensitivity to choice of parameters of the GA/KNN method," Bioinformatics, vol. 17, no. 12, pp. 1131-1142, 2001.

[5] Cho, S.-B. and Ryu, J., "Classifying gene expression data of cancer using classifier ensemble with mutually exclusive features," Proc. of the IEEE, vol. 90, no. 11, pp. 17441753, 2002. 